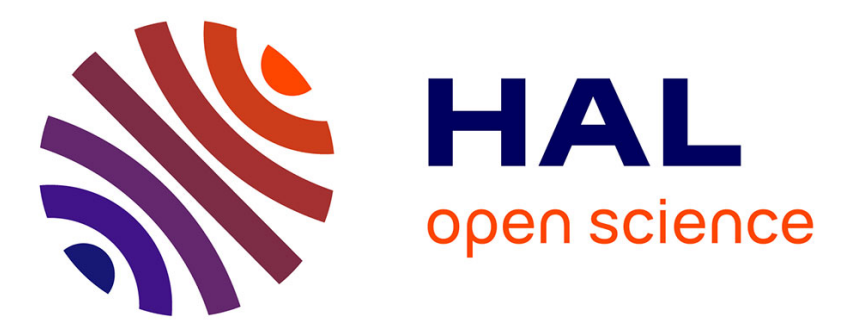

\title{
Yûichi Shionoya, représentant contemporain majeur de la philosophie économique au Japon \\ Gilles Campagnolo
}

\section{To cite this version:}

Gilles Campagnolo. Yûichi Shionoya, représentant contemporain majeur de la philosophie économique au Japon. Revue de Philosophie Economique / Review of Economic Philosophy, 2019, 20 (1), pp.175185. 10.3917/rpec.201.0175 . hal-02365571

\section{HAL Id: hal-02365571 \\ https://hal-amu.archives-ouvertes.fr/hal-02365571}

Submitted on 25 May 2020

HAL is a multi-disciplinary open access archive for the deposit and dissemination of scientific research documents, whether they are published or not. The documents may come from teaching and research institutions in France or abroad, or from public or private research centers.
L'archive ouverte pluridisciplinaire HAL, est destinée au dépôt et à la diffusion de documents scientifiques de niveau recherche, publiés ou non, émanant des établissements d'enseignement et de recherche français ou étrangers, des laboratoires publics ou privés. 


\title{
Yuichi Shionoya, représentant contemporain majeur de la philosophie économique au Japon
}

\author{
Gilles Campagnolo
}

\section{Avant-propos}

Décédé le 25 août 2015, Yuichi Shionoya avait acquis une stature internationale en développant en particulier le champ de la philosophie économique. Nous consacrons une partie documentaire à cet économiste soucieux de la réflexivité au sein de sa discipline dans le présent numéro spécial « Philosophie économique au Japon ». Cet hommage est l'occasion de présenter au public de la Revue de philosophie économiquel Review of Economic Philosophy deux de ses textes inédits en toute autre langue que le japonais. Afin de mieux les situer, nous proposons cette courte présentation en français accompagnée d'une nécrologie en anglais. Nous remercions le professeur émérite Bertram Schefold, qui avait présidé une «table-ronde » d'hommage à Shionoya tenue en 2016 ; son texte constitue également un précieux témoignage de l'interaction entre chercheurs européens et japonais dans notre discipline et de l'influence forte exercée, en l'occurrence dans le cas de Shionoya, par la pensée de langue allemande ${ }^{1}$.

Les textes de Shionoya présentés en traduction française dans le présent numéro thématique sont tirés de son ouvrage Économie et éthique : philosophie de l'État-providence ${ }^{2}$. Le premier texte constitue l'introduction du volume original, le second son appendice de nature historique et philosophique à la fois. Dans ce texte qui sert à la fois de conclusion au volume, la dernière phrase exprime tout l'esprit de la quête de Shionoya, dans ses propres termes : « dans ce livre, nous avons tenté de remplir consciencieusement et méthodiquement notre devoir consistant à rapporter la dimension des idées à celle des systèmes ». C'était l'esprit de la tâche qu'il avait poursuivie à travers la philosophie économique et c'en était, en somme, à ses yeux, la définition. Il l'indique également dans le premier texte, "L'économie, d'une rive à l'autre », où il file la métaphore de ses vues : "ce livre poursuit le but de faire réfléchir au système de l'État-Providence dans le cadre académique large des rapports entre économie et éthique ». Il était impossible de traduire l'ouvrage en entier, mais la présentation du plan, donnée à la fin du premier texte, fournira au lecteur une idée générale assez indicative de l'entreprise en son entier.

À propos de ces deux textes, jusqu'ici inédits en toute autre langue que leur original japonais, disons encore que, dans «L'économie, d'une rive à l'autre », la métaphore du fleuve permet à Shionoya de lier ses visions de l'essence et du rôle de la philosophie économique. L'occasion du propos est la mesure des enjeux et des difficultés de l'État-providence japonais. Ces réflexions, émises en 2002, nous semblent sans doute encore plus d'actualité à l'heure de cette publication, alors que la politique économique du Premier ministre Shinzô Abe, connue sous le nom d' "Abenomics » suscite le débat quant aux résultats qu'elle a donnés comme aux limites qu'elle a atteintes.

\footnotetext{
${ }^{1}$ Un exemple parmi tant d'autres de cette coopération est notamment la conférence tenue à Berlin en 2008 sur cette influence («Der Einfluss Deutschsprachigen Wirtschaftswissenschaftlichen Denkens In Japan »). Elle a donné lieu au vol. 115/XXVII dans la collection «Schriften des Vereins für Socialpolitik Gesellschaft für Wirtschafts- und Sozialwissenschaften des Studien zur Entwicklung der Ökonomischen Theorie ». La conférence fut l'occasion de présenter la liste des archives en langue allemande des bibliothèques universitaires au Japon que nous avions dressée : G. Campagnolo, «Liste Deutscher und Österreichischer Archive von Sozialwissenschaftlern in universitären Sondersammlungen in Japan », publiée par Heinz Kurz (Dir.) dans le volume susdit, p. 131-177. ${ }^{2}$ Tokyo, Presses universitaires de Tokyo, 2002, traduction par Kaori Kasaï (docteure en lettres modernes de l'université Diderot-Paris VII), avec l'aimable autorisation des éditions de l'université de Tokyo et de la fille de Yuichi Shionoya, que nous remercions ici vivement.
} 
Le second texte a été sélectionné, d'une part, pour sa valeur documentaire quant à l'histoire de la philosophie économique au Japon, dont il évoque le moment fondateur puisque Shionoya y retrace le parcours et les idées des deux fondateurs de la philosophie économique au Japon: Kiichirô Sôda et Kôzô Sugimura, et, d'autre part, pour ses dimensions d'épistémologie et de théorie de la connaissance, où se manifestent très significativement l'importance de la philosophie de langue allemande dans le développement de la philosophie économique au Japon, Erkenntnislehre et néo-kantisme en particulier. C'est toute une époque que Shionoya fait revivre dans ce texte ${ }^{3}$; mais c'est aussi une orientation quant aux exigences pour un renouveau de la philosophie économique au Japon. Celui-ci pourrait s'opérer dans la perspective de ce que Shionoya appelle un «idéalisme » en mesure de répondre aux exigences du présent, alors que les théories économiques contemporaines expriment elles-mêmes le besoin de se revivifier dans la convergence des idées et des systèmes que Shionoya évoque. Il écrit : «De nos jours, la philosophie économique doit synthétiser (ou globaliser) les théories du "bien", de la "justice" et de la "vertu" ; cela signifie que nous sommes parvenus à la jonction entre les deux courants principaux de l'histoire des méthodes en économie ».

Un dossier documentaire pour un auteur décédé est une innovation dans la Revue de philosophie économique que la personnalité et le rôle de Yuichi Shionoya comme représentant contemporain majeur de la philosophie économique au Japon justifie amplement pour nos lecteurs. Les pages qui suivent retracent, en guise d'introduction, sa vie et son œuvre.

\section{Présentation de la vie et de l'auvre de Yuichi Shionoya}

Né en 1932 dans la préfecture de Kôchi (île de Shikoku, Ouest du Japon), Yuichi Shionoya était d'une famille de lettrés. Son père, Tsukumo, est connu pour avoir traduit en japonais dès 1939 la Théorie générale de Keynes. Le jeune Yuichi, formé en sciences économiques ${ }^{4}$ à l'université de Nagoya (l'une des sept anciennes universités «impériales »), devint en 1972 professeur titulaire de l'université Hitotsubashi. Il devait assumer plus tard la présidence de cette université (1988-1992), ainsi que la vice-présidence (1991-1992) de l'association regroupant les universités d'État, dans un pays où les universités publiques d'État, rivalisent avec celles dépendant des municipalités et les universités privées (dont certaines ont les principales facultés de sciences économiques japonaises, comme l'université Keio). La concurrence qui règne impose à ces universités de briller dans la recherche afin d'attirer les meilleurs étudiants, et ce travail difficile de chercheur trouva un exemple frappant de réussite internationale avec Shionoya, qui contribua à la renommée mondiale de l'université Hitotsubashi ${ }^{5}$.

\footnotetext{
${ }^{3}$ Donnons ici un seul un exemple de ces événements des années 1920 auxquels Shionoya fait allusion dans son texte et qu'il est impossible de retracer tous. Sugimura écrivit sa thèse de doctorat sur les Recherches sur la théorie de la valeur dans la société et en économie : elle fut rejetée, provoquant un scandale connu sous le nom du « vote blanc » (il avait obtenu seulement 13 votes sur 21 , alors qu'il fallait $3 / 4$ du corps électif choisi selon les règles prévalant alors). Sugimura enseignait déjà à l'université Hitotsubashi. De rage, il voulut abandonner, malgré les objurgations du président de l'université, qui annula la décision du jury et avalisa sa thèse. Mais Sugimura refusa. La polémique s'enflamma et divisa l'université, suscitant des démissions en chaîne, dont celles du président et de Sugimura lui-même. Sur le conseil du successeur à la présidence de l'université, Sugimura préféra publier son texte chez un éditeur indépendant, en apportant quelques modifications. En 1938, alors qu'il travaillait à la Chambre de commerce de Shanghaï (occupée par le Japon), il fut finalement reçu docteur pour une autre thèse : Structure de l'éthique économique. Après-guerre, il redevint enseignant à Hitotsubashi. Il mourut en 1948. L'on dit parfois que nombre de ses idées inspirèrent le Premier ministre japonais Oohira (en charge de 1978 à 1980).

${ }^{4}$ En japonais 経済学 (keizaïgaku) désigne la science économique d'origine occidentale au sens le plus strict, selon le terme formé lors de l'introduction de la discipline au Japon : voir l'introduction générale de ce numéro.

${ }^{5}$ L'université Hitotsubashi s'appela d'abord « École supérieure de commerce de Tokyo », puis « université du commerce de Tokyo » avant de prendre le nom sous lequel elle est aujourd'hui connue, lors de son déménagement
} 
Ces recherches portaient en particulier sur la philosophie économique, comprise comme l'étude des valeurs et des questions fondamentales dans l'agencement de la logique économique et des questions morales et sociales. Elles requéraient un matériau que l'histoire de la pensée économique pouvait lui fournir. Il contribua à éclairer ainsi, d'une part, l'histoire du développement des sciences économiques au Japon même, et d'autre part, les sources d'influence majeures qui avaient contribué à cet essor au Japon, tout particulièrement les sources allemandes: Shionoya devint un spécialiste reconnu de l'École historique du dixneuvième siècle, ainsi que de Max Weber et de Josef Schumpeter. Il publia à leur sujet et collabora éminemment au sein de la profession au plan international ${ }^{6}$.

Ces centres d'intérêt impliquaient et expliquaient la grande attention qu'il manifestait envers les doctrines institutionnalistes et welfariste : la théorie du bien-être touchait aux enjeux de l'État-providence, qui le faisaient intervenir dans les débats académique international et politique national, touchant le cas spécifique du Japon. Sa reconnaissance à ces deux niveaux lui valut d'occuper un certain nombre de positions d'expertise nationale comme, de 1995 à 2000, la direction de l'Institut national des enquêtes démographiques et de la protection sociale.

Shionoya publia beaucoup et notamment en anglais. Citons parmi ses titres les plus connus à l'étranger: Schumpeter and the Idea of Social Science: A Metatheoretical Study (Cambridge University Press, 1997, réédité en 2007), The Soul of the German Historical School. Methodological Essays on Schmoller, Weber, and Schumpeter (Springer, 2005), Economy and Morality. The Philosophy of the Welfare State (Edward Elgar, 2005) ${ }^{7}$. Les ouvrages parus en anglais sur Schumpeter, donc, et sur l'école historique allemande en général, lui ont valu la reconnaissance internationale, notamment dans la communauté des économistes allemands.

Mais il convient de souligner pour le public ne parlant pas japonais que sa production dans cette langue, évidemment trop longue pour la rappeler en entier ici, avait un rôle d'orientation dans les débats académique et politique nippons. En particulier, en ce qui concerne la «philosophie économique au Japon », deux de ses ouvrages japonais Philosophie économique : une approche herméneutique (2009) et celui choisi pour les deux textes du présent numéro: Économie et éthique : philosophie de l'État-providence (2002) ont-ils contribué au renouveau de ce champ disciplinaire surgi dès les années 1920 dans les travaux de Sôda et de Sugimura.

De nature plus théorique, un autre ouvrage, dont le titre se traduirait par Current Prices Problems ou Le coût de la vie dans le monde contemporain ${ }^{8}$ fait figure de classique dans sa langue originale sur le sujet de la monnaie. En outre, Shionoya accordait une grande importance aux archives présentes à l'université Hitotsubashi où il organisa un « Fonds Schumpeter » et où le «Centre pour l'histoire de la littérature des sciences sociales occidentales » abrite de nombreuses collections, dont celle du fondateur de l'école autrichienne d'économie, Carl Menger? .

vers la grande banlieue ouest de la capitale, dans la ville de Kunitachi. Le sens de son nom, qui signifie « un seul pont », rappelle le pont qui reliait directement son ancien emplacement au palais impérial au centre de Tokyo. Cette université fut également celle de Sôda et de Sugimura dont Shionoya narre le rôle fondateur pour la philosophie économique japonaise.

${ }^{6}$ Concernant les publications, voir la bibliographie de ses œuvres en langues autres que le japonais à la fin de cette présentation. Un exemple de coopération internationale significatif est qu'en 1986 Shionoya fut l'un des cofondateurs de la société savante «International Joseph A. Schumpeter Society » et qu'il la présida de 1990 à 1992. Il organisa notamment en 1992 à Kyoto un colloque qui fit date sur l'auteur.

${ }^{7}$ Références de la liste complète des ouvrages de Shionoya en langue anglaise en fin de présentation. Il n'existe de publication en langue française à notre connaissance que les textes fournis dans le présent numéro. Les références aux publications en japonais sont données autant que de besoin en notes de bas de page des deux textes traduits.

${ }^{8}$ Titre de l'ouvrage : «現代の物 価》(Gendai no Bukka), Tokyo, Nihon Keizai Shinbunsha, 1973.

${ }^{9}$ Voir (en français) G. Campagnolo, «Un exemple de réception de l'économie occidentale au Japon : le Fonds Carl Menger de l'université Hitotsubashi », in Lucas N. et Sakai C. (dir.), Japon Pluriel 4, publié par la Société 
On l'aura compris, Shionoya fut un personnage très honoré. En 1991, il reçut le Prix de l'Académie du Japon. ${ }^{10}$ Ce prix lui fut attribué pour son œuvre et, expressément, pour ses travaux en philosophie économique, en particulier sur les systèmes conceptuels touchant la notion de valeur ${ }^{11}$. Shionoya y met en contraste les principes respectifs de plusieurs doctrines quant aux conceptions de la justice et de la protection sociales et il passe en revue les effets institutionnels de leur mise en application. Il s'inspire notamment d'une lecture interprétative personnelle de A Theory of Justice de John Rawls. En 2002, Shionoya reçut également le prix de la «personne de mérite culturel $»^{12}$.

Le dernier ouvrage publié par Shionoya (La pensée économique du romantisme dans les arts, en éthique et dans l'histoire, 2012, en japonais) témoigne de son intérêt pour cette autre tradition, la britannique, de laquelle son enquête sur les rapports entre keynésianisme et institutionnalisme moderne l'avait rapproché. Ses derniers articles concernaient ainsi la pensée de John Ruskin. Il y argumentait que la perspective de l'école dite «d'Oxford » représentait, pour la pensée anglaise, un équivalent des vues de Sozialpolitik présentes dans l'école historique allemande, notamment en raison de l'orientation dite «éthique » au sein de cette dernière ${ }^{13}$. À la fin de sa vie, Shionoya s'intéressa ainsi à T. H. Green, l'héritier anglais du hégélianisme, à Ruskin et à Hobson dans le volume collectif No Wealth but Life; Welfare economics and the welfare state in Britain (dirigé par Backhouse et Nishizawa, 2010) ${ }^{14}$. Le dernier texte (posthume) de Shionoya consiste en un article resté inachevé qui trouve son origine dans la communication qu'il avait donnée devant la « Keynes Society in Japan $»^{15}$.

Si l'on voulait résumer l'éthique que Shionoya proposait au final comme sa propre contribution au renouveau de la «philosophie économique » qu'il appelait, il serait sans doute juste d'agencer les trois éléments suivants en un système (que nous ne tenterons cependant pas de formuler, ce qui exigerait une toute autre ampleur que le cadre d'une simple présentation) :

Premièrement, Shionoya pense une théorie morale fondée sur l'utilité, une théorie du bien touchant l'action individuelle et les activités privées (chez des auteurs utilitaristes comme Bentham ou John Stuart Mill et chez les subjectivistes comme Menger);

Deuxièmement, il propose une théorie morale fondée sur les droits afin d'établir des critères et des règles de justice (sur le modèle des philosophies de Kant et des auteurs néokantiens, d'une part, de Rawls et des théories contemporaines, d'autre part) ;

Française des Études japonaises, Paris, Philippe Picquier, 2001, p. 211-221. Une version japonaise est parue (trad. Kôichi Yamazaki) 《メンガ一文庫・ある経済思想の原資料 (Mengâ-bunko: aru keizaishisô no genshiryô) », Bulletin of the Center for Historical Social Science Literature, de mars 2002, 22, p. 23-39

${ }^{10}$ Le prix 学士院 (Gakushiinshô) est le plus prestigieux prix du monde académique japonais. Il est délivré annuellement par l'Académie du Japon, l'équivalent de l'Académie des sciences morales et politiques en France.

${ }^{1111}$ En témoigne notamment la recension 価值理念の構造一効用対権利 (Kachirinen no Kôzô - Kôyô Taikenri) Tokyo, Iwanami Shoten, 1986, par Tsuneo Ishikawa, revue Keizai Kenkyû (Recherches économiques, de l'éditeur Iwanami Shoten, à Tokyo - la précision s'impose car il y a plusieurs revues homonymes), 37, p. $276-278$.

${ }^{12}$ Le prix 文化功労者 (Bunka Kôrosha) consiste en pratique dans le second prix culturel le plus important au Japon, celui que peuvent recevoir des écrivains de fiction ou des acteurs du théâtre traditionnel, voire des sportifs de grand renom. Le lecteur français en mesurera l'importance au fait que c'est le prix que reçut, en son temps, Junichiro Tanizaki, le seul écrivain japonais à figurer à ce jour dans la «collection de la Pléiade ».

${ }^{13}$ Voir de Shionoya l'article «Ruskin's romantic triangle: neither wealth nor beauty but life », History of Economic Ideas, 2014/1, p. 15-50.

${ }^{14}$ Shionoya, Y (2010) «The Oxford approach to the philosophical foundations of the welfare state » in Roger E. Backhouse et T. Nishizawa (dir.), No Wealth but Life: Welfare Economics and the Welfare State in Britain (18801945), Cambridge University Press, 2010, p. 91-113. Une version japonaise de ce texte parut dans la revue Keizai Kenkyu (Recherches économiques, de l'éditeur Iwanami Shoten, à Tokyo) en avril 2014 (65, p. 113-125)

${ }^{15}$ Ce texte est publié dans le volume (en japonais) dirigé par Nishizawa et Hirai en 2016 dans le cadre d'un projet sur les économistes de Cambridge : «Philosophy, Arts, Economics of Keynes : in the light of Enlightenment vs. Romanticism ». 
Troisièmement, il veut voir advenir une théorie de la vertu définie en rapport aux capacités (voire aux capabilités, si l'on reprend le terme anglais d'un autre auteur nonoccidental de par son origine, Amartya Sen - Shionoya le reprend toutefois très peu); il considère les existences individuelles avant tout (et renvoie amplement à l'usage que les penseurs allemands firent d'Aristote, comme aux idées d'un néo-hégélien anglais comme Green). Ces trois dimensions fournissent le repère des positions que Shionoya conjuguait.

La coordination de ces systèmes éthiques potentiels dans une philosophie «à trois branches » que ses recherches tendent à unifier exigerait en soi une analyse des objets que Shionoya a sélectionnés pour en donner l'évaluation morale. Shionoya a choisi sa terminologie des valeurs fondamentales, en étant lui-même, comme Sôda et Sugimura, profondément inspiré par les écoles allemandes, et il fut conscient de l'importance de souligner leur caractère opérationnel car une action efficace en vue du « bien » requiert un droit fondé sur des règles de justice - ou, dit autrement, la justice fondée sur des règles institutionnelles de droit exige une conduite visant l'excellence en suivant la vertu de l'être, compris comme agent économique ${ }^{16}$.

Mais, une fois le vocabulaire posé (selon des sources d'inspiration qui sont rarement celle des économistes contemporains, reconnaissons-le), quel ordre de priorité adopter entre ces trois dimensions ? Dans sa quête admirable d'une théorie compréhensive ${ }^{17}$ de la vie, comprise «pleinement» comme nature humaine « intégrale », Shionoya explora différentes possibilités dans ses œuvres au long de sa carrière. Cette caractérisation doit rappeler deux points essentiels qui peuvent servir de guides que le lecteur pourra, disons-le pour conclure, garder présents à l'esprit. Ce sont les suivants.

D'une part, Shionoya avait forgé ses vues, sur le romantisme comme sur les Lumières, dans un long apprentissage et une pratique approfondie des thématiques germaniques, allemandes (Rickert, Weber) et autrichiennes (Menger, Schumpeter). Il était au Japon l'un des meilleurs connaisseurs de ces auteurs, au moins quant à la facette économique de leurs œuvres ${ }^{18}$. Les textes présentés en traduction dans ce numéro illustrent au plus haut point cette riche connaissance de la philosophie et de l'analyse éthico-historique allemandes, mise au service d'une conceptualisation des tâches de la philosophie économique non seulement au Japon, mais encore à titre général.

Cela nous mène au second point, car d'autre part, Shionoya tirait de cette inspiration par les écoles allemandes les éléments d'une critique méthodologique précise des positions de l'économie dite "néoclassique » bornée par son attention exclusive à l'utilitarisme sous ses formes diverses. Shionoya était un penseur indéniablement critique, tout en jouant un rôle éminent au sein des institutions. Se tenant au courant de l'apport de l'économétrie modélisatrice, il jugeait qu'une «good society » devait se pourvoir également, tout particulièrement dans le contexte nippon, d'une éthique de la vertu adaptée au monde contemporain pour servir de fondement solide à une économie conservant le sens du droit (de la justice) et améliorer la qualité de vie en pratique, dans une société dont l'économie post-industrielle connaît les difficultés d'une population vieillissante, voire déclinante. La conservation matérielle est le souci pragmatique des Japonais, y compris des «philosophes économiques » japonais.

Dans cette perspective, laissons le dernier mot à Shionoya Il avait choisi la citation suivante de son auteur de prédilection, Schumpeter (tirée d'un article du Harvard Crimson d'avril 1944), pour son propre dernier article, et ce choix dit la philosophie qui inspirait à son

\footnotetext{
${ }^{16}$ Le texte de Bertram Schefold qui suit peut se lire comme une tentative d'articuler ces dimensions dans la pensée de Shionoya, au regard de l'influence exercée par les pensées des auteurs de langue allemande.

$17 \mathrm{Au}$ sens de verstehende, en allemand là encore, c'est-à-dire en conjuguant l'entendement et le souci systématique d'englober unitairement les disciplines au sein de la connaissance dans les sciences sociales

${ }^{18}$ Citons également à cet égard Kiichiro Yagi, qui fait l'honneur de donner un article dans le présent numéro, et dont la renommée internationale n'avait rien à envier à celle de Shionoya, en particulier concernant la connaissance des textes et des archives de Menger (notamment, mais pas seulement, celles conservées au Japon).
} 
tour Shionoya : « une philosophie inhabituelle de la vie [...] une idée de la vie abondante et englobante qui comprend l'économie, la philosophie, la politique, la science, l'art et l'amour » Shionoya commentait cette citation en disant que Schumpeter exprimait ce qui guidait son programme de recherche, que l'on pouvait qualifier (dans une veine wébérienne) de « sociologie compréhensive (allemande) ${ }^{19}$.

C'est un programme sur tant de points similaire qui inspirait le représentant majeur de la philosophie économique japonaise que fut excellemment Yuichi Shionoya.

\section{Références des monographies et des ouvrages dirigés par Shionoya en langue anglaise}

NB : Cette liste n'est pas une bibliographie exhaustive ; elle n'inclut ni les chapitres d'ouvrages, ni les nombreux articles publiés par Shionoya. Il n'existe à notre connaissance aucun ouvrage de Shionoya traduit en langue française à cette heure.

Par ailleurs, les deux textes traduits dans le présent volume sont des inédits en toute langue autre que le japonais. La Revue de philosophie économique/Review of Economic Philosophy remercie la maison d'édition et les ayant-droits de Y. Shionoya.

1. 1993 (avec Peter Koslowski) (dir.) The Good and the Economical: Ethical Choices in Economics and Management (coll. Studies in Economics, Ethics and Philosophy), Berlin et New York: Springer.

2. 1994 (avec Mark Perlman) (dir.) Schumpeter in the History of Ideas (en japonais et en anglais), éd. par International Schumpeter Society pour la version anglaise. Ann Harbor: University of Michigan Press.

3. 1994 (avec Mark Perlman) (dir.) Innovation in Technology, Industries, and Institutions: Studies in Schumpeterian Perspectives. Ann Harbor: University of Michigan Press.

4. 1997. Schumpeter and the Idea of Social Science: A Metatheoretical Study, Cambridge University Press (rééd. coll. Historical Perspectives on Modern Economics, 2007).

5. 2000. The German Historical School: The Historical and Ethical Approach to Economics, Abingdon et New York : Routledge, coll. Studies in the History of Economics.

6. 2000 (avec Kiichiro Yagi). Competition, Trust, and Cooperation: A Comparative Study (Ethical Economy), New York: Springer.

7. 2005. The Soul of the German Historical School: Methodological Essays on Schmoller, Weber and Schumpeter, New York: Springer, coll. The European Heritage in Economics and the Social Sciences.

8. 2005. Economy and Morality: The Philosophy of The Welfare State, Cheltenham: Edward Elgar.

9. 2008 (avec Tamotsu Nishizawa) (dir.) Marshall and Schumpeter on Evolution: Economic Sociology of Capitalist Development, Cheltenham: Edward Elgar.

\footnotetext{
${ }^{19}$ Texte original: « an unusual philosophy of life [...] an idea of rich and full life to include economics, politics, science, art, and love » J. A. Schumpeter, Harvard Crimson, 11/4/1944, cité par Shionoya dans son texte pour l'ouvrage No Wealth but Life; Welfare economics and the welfare state in Britain, op. cit., 2010, p. 267.
} 